\title{
Impedance-Based Modelling of Hybrid AC/DC Grids With Synchronous Generator for Interaction Study and Dynamic Improvement
}

\author{
Adedotun J. Agbemuko ${ }^{\mathrm{a}, \mathrm{b}, *}$, José Luis Domínguez-García ${ }^{\mathrm{a}}$, Oriol Gomis-Bellmunt ${ }^{\mathrm{b}}$
}

\begin{abstract}
${ }^{a}$ IREC Catalonia Institute for Energy Research, Jardins de les Dones de Negre 1, 2a. 08930 Sant Adrià de Besòs, Barcelona, Spain
${ }^{b}$ Department of Electrical Engineering, Universitat Politècnica de Catalunya UPC, Av. Diagonal, 647, Pl. 2. 08028 Barcelona, Spain
\end{abstract}

\begin{abstract}
Hybrid AC/DC grids are increasingly been seen as the most important network structure for future transmission infrastructures. An emerging challenge to such grids is the constant interaction between various components in the system. Interactions could occur in many intricate forms including between and within the AC and DC subnetworks. However, considering the complexities involved, methods for detection that are intuitive, compact, and relatively straightforward to apply are lacking. In this paper, device-level closed-loop impedance models of subsystems in a hybrid AC/DC grid are extended compactly to subnetwork equivalents in the form of matrices. Then, interaction analysis between a voltage source converter (VSC) interconnected on both sides, and a synchronous generator with excitation control is carried out as a case study to highlight potentially detrimental interactions. The analysis is carried out in the frequency domain and validated with a nonlinear time domain simulations in MATLAB/SIMULINK.
\end{abstract}

Keywords: VSC, Synchronous generator, Impedance-based analysis, Interactions, HVDC, hybrid AC/DC grid

\section{Introduction}

Power system literature on emerging topics over the last decade has been offering views on the potential structure of future power systems, considering the impact of renewable energy integration, and the changing energy landscape [1]. A general consensus in the power systems community is the expected role of DC circuits and systems. More so, DC systems buoyed by power electronic components will play a huge role in sustainable energy systems [2-4].

The rapid advances in the field of power electronics have accelerated the potentials for HVDC transmission based on voltage source converter (VSC) technology, that potentially allows for the forming of multi-terminal DC networks [5, 6]. Therefore, future infrastructures are expected to leverage both $\mathrm{AC}$ and $\mathrm{DC}$ characteristics on a large-scale. Hence, operation would involve new and existing AC networks, potentially with synchronous generation and their controls present [7]. This would inevitably lead to interactions between the converter and its controls, and that of synchronous generation.

Literature studying interactions of hybrid AC/DC systems have mainly focused on one side of the converter - $\mathrm{AC}$ or DC, with very few focusing on both $\mathrm{AC}$ and DC grids [8-11]. Furthermore, most analysis have been based on traditional modal analysis for conventional AC

\footnotetext{
* Corresponding author

Email address: aagbemuko@irec.cat (Adedotun J. Agbemuko)
}

grids which rely mainly on state-space approaches. However, VSC converter technologies are not yet mature compared to the line commutated converter (LCC), thus, manufacturers are wary of explicit information sharing that is required to re-tune these devices. It is also well-known that state-space approaches do not support independent redesign or re-tuning of controllers. Moreover, with the expected domination of power electronic converters, these methods are not sufficient to provide adequate information about system dynamics in the presence of these devices [12].

The impedance modelling method is re-emerging to cater for the peculiarities with VSC technologies [13-15]. This methodology has gained significant attention in recent times and has been widely applied to converter dominated power systems to analyse stability amongst other issues [16-19]. The impedance modelling methodology has been further extended to analyse the resonance and stability issues specific to wind power plants connected via converters and real experiences have highlighted critical interactions between wind farms controls and HVDC converters [20-23]. Whereas, [24] derived the analytical impedance equivalents for high frequency analysis, however without the consideration of synchronous generation. Impedance models have been well-applied to stability analysis, but not interaction analysis and dynamic responses considering hybrid AC/DC grids.

In this paper, the mechanism of interactions between the DC and AC subnetworks that includes a controlled synchronous generator and a grid connected VSC is anal- 


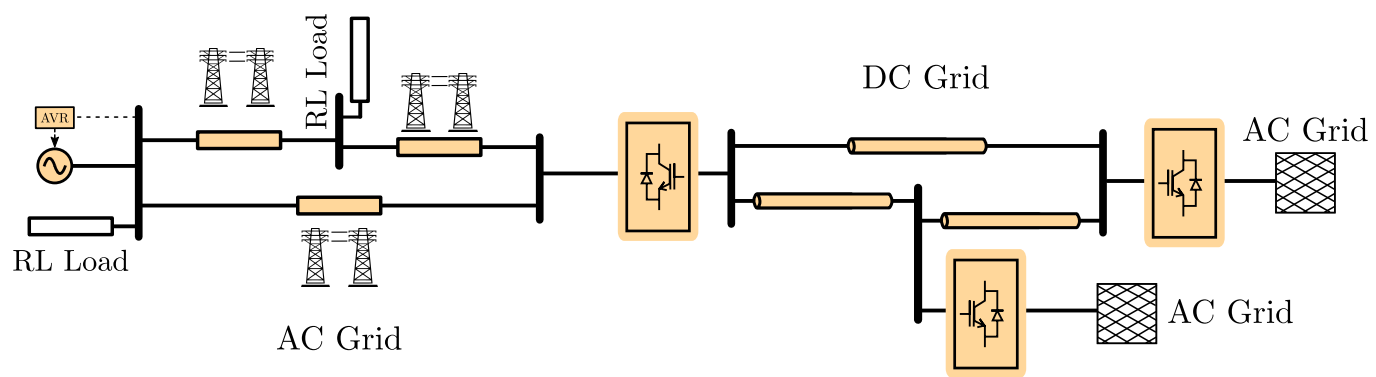

Figure 1: Single-line diagram of a hybrid AC-DC grid

ysed. First, the entire network is decomposed into the distinct subnetworks, and subnetworks further decomposed into key subsystems. Then, the equivalent model of each subsystem is derived, and the potential conflicts of control requirements between subnetworks through the common interface are highlighted through a sensitivity analysis. Subsequently, the subsystems were interconnected in the structure of each subnetwork. Given the decoupled equivalent models of each subnetwork in the form of closed-loop impedance matrices, interactions between both subnetworks through the VSC is analysed. Finally, it is shown that a compromise between controller parameters of the VSC and synchronous generator is required for an acceptable performance in both subnetworks.

The rest of this paper is organized as follows; Section 2 derives the impedance models of all identified subsystems in the grid, Section 3 focuses on the interconnection and aggregation of the subsystems in each subnetwork to form the system, Section 4 discusses a case study of the described methodology, and conclusions in Section 5.

\section{Impedance-Modelling of Subsystems}

\subsection{Overview of System and Modelling Framework}

Impedance is a fundamental property of the physical power system and its corresponding electrical circuit. In this section, the feedback input-output impedance of each subsystem is derived in Laplace's domain ( $s$-equivalents). In this paper, each representative impedance model is obtained from the device-level expressions. The final aim is to obtain the equivalent input-output representation of each subsystem. Fig. 1 depicts the simplified one-line diagram of a VSC coupled hybrid AC/DC grid, showing the network topology and main subsystems. The AC side consists of a synchronous generator with its excitation system, two $R L$ loads, transmission lines, and a VSC interface. Whereas, the DC side consists of three VSCs in different control modes and an interconnecting network of cables, with aggregated AC sides of two VSCs for simplicity.

For any active device - converters, generators etc. injecting currents, imposing voltages, or related variables on the network at terminal $i$, the linearized equations can be expressed as

$$
\Delta \dot{x}_{i}=\mathbf{A}_{i} \Delta x_{i}+\mathbf{B}_{i} \Delta u_{i}
$$

$$
\Delta Y_{i}=\mathbf{C}_{i} \Delta x_{i}+\mathbf{D}_{i} \Delta u_{i}
$$

where $\Delta x_{i}$ is the linearized state vector of each active device, $\Delta u_{i}$ is in general the vector of input terminal voltages and/or currents relevant to each active device, and $\Delta Y_{i}$ is the output vector, whereas matrices $\mathbf{A}_{i}, \mathbf{B}_{i}, \mathbf{C}_{i}$, and $\mathbf{D}_{i}$ are matrices of appropriate dimensions. For VSCs that concurrently inject currents and impose voltages into the two subnetworks (AC/DC), $\Delta u_{i}$ and $\Delta Y_{i}$ consists of both voltages and currents corresponding to the $\mathrm{AC}$ and $\mathrm{DC}$ sides as will be shown in subsequent sections. Whereas, for synchronous generators, $\Delta u_{i}$ and $\Delta Y_{i}$ consists of either one of voltages and currents respectively (at a circuit-level) or vice-versa.

Expressing (1a) in Laplace domain, and substituting for $\Delta x_{i}$ from the resulting expression into (1b)

$$
\frac{\Delta Y_{i}}{\Delta u_{i}}=\mathbf{C}_{\mathbf{i}}\left(s \mathbf{I}-\mathbf{A}_{\mathbf{i}}\right)^{-1} \mathbf{B}_{\mathbf{i}}
$$

where $\mathbf{I}$ is the identity matrix, and $\mathbf{D}_{i}=0$. The expression on the right hand side of (2) is a matrix with all the inputoutput transfer functions of each active device. Therefore, all subsystems will be modelled with a view towards the expressions in the form of equation (2).

\subsection{Grid Connected VSC Input-output dq Impedance}

Fig. 2 depicts a simplified single-line diagram of a VSC station with the typical control block layout. A combination of control strategies and alternative schemes can be applied to the VSC as required. In this paper, the VSC is modelled considering four distinct controllers, the DC voltage, AC voltage, active power, and droop controllers. However, there are three combinations that may be applied in pairs with possibility to switch - the DC and $\mathrm{AC}$ voltage, active power and $\mathrm{AC}$ voltage, droop and $\mathrm{AC}$ voltage control modes.

\subsection{1. $A C$ and DC Voltage Controlled VSCs}

The outer AC and DC voltage control blocks consists of proportional and integral (PI) type compensators denoted by $K_{u}(s)$ and $K_{v}(s)$ respectively. Generically, modelling the converter as a subsystem, first without connection to the grid, the open-loop dynamic circuit equations 


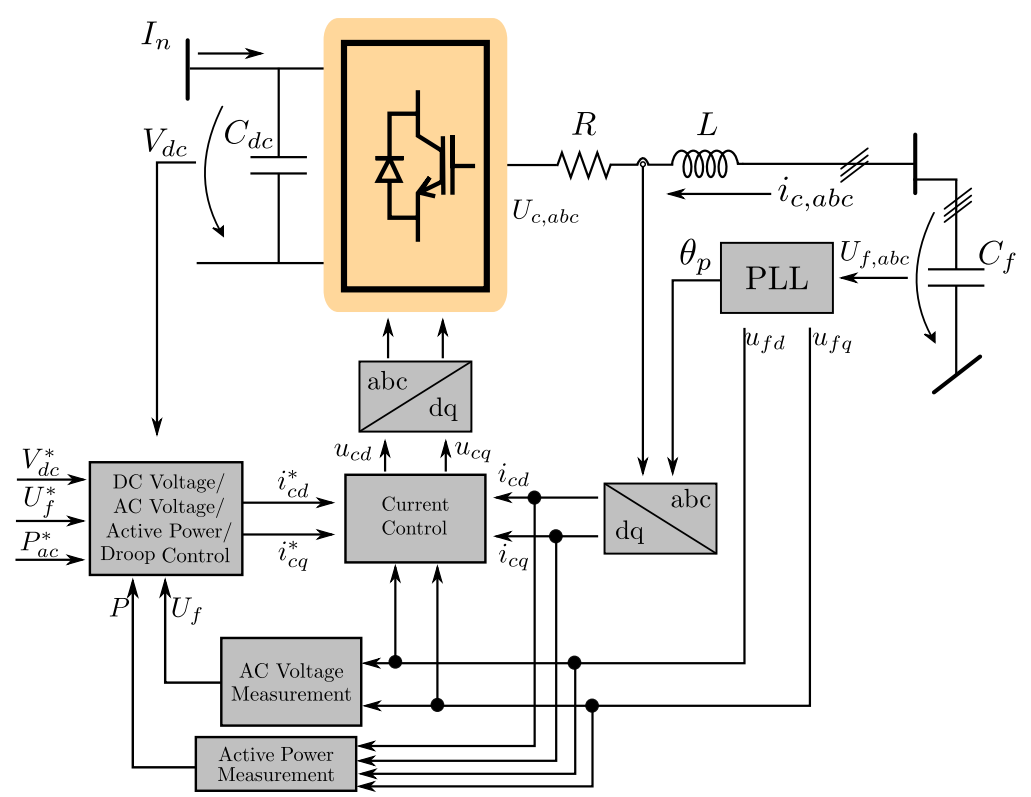

Figure 2: Schematic diagram of a generic VSC station and control blocks

of the physical system on each side of the VSC, in the synchronous reference frame are described compactly by

$$
\begin{gathered}
L \frac{d i_{c d, q}}{d t}=-R i_{c d, q}-\omega_{1} L i_{c q, d}-u_{c d, q}+u_{f d, q} \\
C_{d c} \frac{d V_{d c}}{d t}=\frac{P_{a c}}{V_{d c}}+I_{n}
\end{gathered}
$$

where $i_{c d, q}$ are the converter currents flowing from the point of common coupling (PCC) on either axes; likewise, $u_{c d, q}$ are the converter voltages on the $\mathrm{AC}$ side, $u_{f d, q}$ are the PCC voltages, $V_{d c}$ is the DC terminal voltage, $P_{a c}$ is the power injected into the $\mathrm{AC}$ grid, $I_{n}$ is the current flowing through from the DC grid, and $\omega_{1}$ is the AC grid frequency. The passive elements, $R, L$, and $C_{d c}$ are the filter resistance, the sum of filter and transformer inductances, and the equivalent DC capacitance respectively. Linearizing (3) around an operating point, the state-space open-loop equations can be expressed as

$$
\begin{gathered}
\frac{d \Delta i_{c d, q}}{d t}=-\frac{R}{L} \Delta i_{c d, q}-\omega_{1} \Delta i_{c q, d}-\frac{\Delta u_{c d, q}}{L}+\frac{\Delta u_{f d, q}}{L} \\
\frac{d \Delta V_{d c}}{d t}=\frac{\Delta P_{a c}}{C_{d c} V_{d c}^{0}}-\frac{P_{a c}^{0}}{C_{d c}\left(V_{d c}^{0}\right)^{2}} \Delta V_{d c}+\frac{\Delta I_{n}}{C_{d c}}
\end{gathered}
$$

and the PCC voltage is aligned with the $d$-axis. Then, during dynamic changes,

$$
\left\{\begin{array}{l}
\Delta P_{a c}=\frac{u_{f d}^{0} \Delta i_{c d}+i_{c d}^{0} \Delta u_{f d}+i_{c q}^{0} \Delta u_{f q}+u_{f q}^{0} \Delta i_{c q}}{k} \\
\frac{P_{a c}^{0}}{\left(V_{d c}^{0}\right)^{2}}=\frac{I_{d c}^{0}}{V_{d c}^{0}}
\end{array}\right.
$$

where $\left(^{0}\right)$ terms are linearization points, $I_{d c}$ is the converter injection current balanced with the $\mathrm{AC}$ side, and $k=2 / 3$ is the power constant. Substituting (5) into (4b)

$$
\begin{gathered}
\frac{d \Delta V_{d c}}{d t}=\frac{u_{f d}^{0}}{C_{d c} V_{d c}^{0}} \Delta i_{c d}+\frac{i_{c d}^{0}}{C_{d c} V_{d c}^{0}} \Delta u_{f d}+\frac{i_{c q}^{0}}{C_{d c} V_{d c}^{0}} \Delta u_{f q} \\
+\frac{u_{f q}^{0}}{C_{d c} V_{d c}^{0}} \Delta i_{c q}-\frac{I_{d c}^{0}}{C_{d c} V_{d c}^{0}} \Delta V_{d c}+\frac{1}{C_{d c}} \Delta I_{n}
\end{gathered}
$$

Therefore, (4a) and (6) make up the open-loop linearized dynamic equations for the $\mathrm{AC}$ and $\mathrm{DC}$ terminals of the VSC without any control.

To include control, the converter voltages $\Delta u_{c d, q}$ must be eliminated from (4a) since these are free parameters set by the controller. The converter voltages can be determined from the output of the inner-most controller, given as

$$
\Delta u_{c d, q}^{c}=-K_{c}(s)\left(\Delta i_{c d, q}^{*}-\Delta i_{c d, q}^{c}\right)-\omega L \Delta i_{c q, d}^{c}+\Delta u_{f d, q}^{c} .
$$

where $K_{c}(s)$ is the PI compensator of the inner-loop, $i_{c d, q}^{*}$ are the inner-loop reference currents on either axes, and all variables with superscript 'c' are the corresponding converter frame variables as opposed to grid reference frame variables in previous equations. Therefore, a transformation is required from converter to grid frame before elimination. The phase-locked loop (PLL) is responsible for this transformation. Hence, it also imposes it's dynamics on the system. The PLL is introduced through Park's transformation given as

$$
\mathbf{T}\left(\theta_{p}\right)=k\left(\begin{array}{ccc}
\cos \left(\theta_{p}\right) & \cos \left(\theta_{p}-\frac{2 \pi}{3}\right) & \cos \left(\theta_{p}+\frac{2 \pi}{3}\right) \\
\sin \left(\theta_{p}\right) & \sin \left(\theta_{p}-\frac{2 \pi}{3}\right) & \sin \left(\theta_{p}+\frac{2 \pi}{3}\right) \\
\frac{1}{2} & \frac{1}{2} & \frac{1}{2}
\end{array}\right)
$$

where $\theta_{p}$ is the output of the PLL. Due to the inclusion of the nonlinear PLL output angle, linearization around an 
operating point must be done. The linearized transformation matrix between converter frame and grid frame for the converter voltages, and the linearized inverse transformation matrices for the converter currents and PCC voltages respectively are expressed as

$$
\begin{aligned}
\Delta u_{c d, q} & =\mathbf{T}\left(\Delta u_{c d}^{c}, \Delta u_{c q}^{c}, \Delta \theta_{p}\right)^{T} \\
\Delta u_{f d, q}^{c} & =\mathbf{T}^{v}\left(\Delta u_{f d}, \Delta u_{f q}, \Delta \theta_{p}\right)^{T} \\
\Delta i_{c d, q}^{c} & =\mathbf{T}^{i}\left(\Delta i_{c d}, \Delta i_{c q}, \Delta \theta_{p}\right)^{T}
\end{aligned}
$$

where, $\mathbf{T}, \mathbf{T}^{v}$, and $\mathbf{T}^{i}$ matrices can be found in AppendixA, and $\Delta \theta_{p}$ is the linearized output of the PLL, given as [25]

$$
\left(H_{d \theta}(s), H_{q \theta}(s)\right)\left(\Delta u_{f d}, \Delta u_{f q}\right)^{T}
$$

where $H_{d \theta_{p}}(s)$ and $H_{q \theta_{p}}(s)$ predict the impact of the PLL on the system through the PCC voltages, both described in AppendixA. After application of the transformation and inverse transformation matrices to the corresponding variables,

$$
\Delta u_{c d, q}=-K_{c}(s)\left(\Delta i_{c d, q}^{*}-\Delta i_{c d, q}\right)-\omega L \Delta i_{c q, d}+\Delta u_{f d, q} .
$$

The inner-loop references $\Delta i_{c}^{d q *}$ are obtained from the outer-loop controllers as

$$
\begin{gathered}
\Delta i_{c}^{d *}=K_{v}(s)\left(\Delta V_{d c}^{*}-\Delta V_{d c}\right)=-K_{v}(s) \Delta V_{d c} \\
\Delta i_{c}^{q *}=K_{u}(s)\left(\Delta U_{f}^{*}-\Delta U_{f}\right)=-K_{u}(s) \Delta U_{f}
\end{gathered}
$$

with

$$
\left\{\begin{array}{l}
U_{f}=\sqrt{u_{f d}^{2}+u_{f q}^{2}} \\
\Delta U_{f}=\frac{u_{f d}^{0}}{U_{f}^{0}} \Delta u_{f d}+\frac{u_{f q}^{0}}{U_{f}^{0}} \Delta u_{f q},
\end{array}\right.
$$

and the outer-loop references are assumed constant. Substituting (12) into the split form of (11) and the resulting expressions into the corresponding physical equations in (4a), the formulation with controls is obtained.

For the sake of brevity, the matrix coefficients of (1), $\mathbf{A}_{\mathbf{i}}, \mathbf{B}_{\mathbf{i}}, \mathbf{C}_{\mathbf{i}}$ of the $i^{\text {th }}$ converter equipped with $\mathrm{AC}$ and DC constant voltage controllers can be easily constructed with the described equations, with the state variables, input, and outputs as

$$
\begin{aligned}
\Delta x_{i} & =\left(\Delta i_{c d i}, \Delta i_{c q i}, \Delta V_{d c i}, \Delta \theta_{p i}\right)^{T} \\
\Delta u_{i} & =\left(\Delta u_{f d i}, \Delta u_{f q i}, \Delta I_{n i}\right)^{T} \\
\Delta Y_{i} & =\left(\Delta i_{c d i}, \Delta i_{c q i}, \Delta V_{d c i}\right)^{T}
\end{aligned}
$$

where all variables have been previously defined. Finally, following the procedures described in (2), the input-output impedance model of converter $i$, including both $\mathrm{AC}$ and DC terminals of the converter (and the interactions between them at the converter-level) is obtained as

$$
\Delta Y_{i}=\left(\begin{array}{cc|c}
Y_{d d i}(s) & Y_{d q i}(s) & H_{d c i}^{d i}(s) \\
Y_{q d i}(s) & Y_{q q i}(s) & H_{d c i}^{q i}(s) \\
\hline H_{d d i}(s) & H_{q q i}(s) & Z_{d c i}(s)
\end{array}\right)\left(\begin{array}{c}
\Delta u_{f d i} \\
\Delta u_{f q i} \\
\Delta I_{n i}
\end{array}\right)
$$

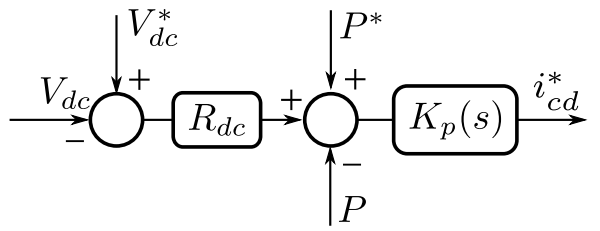

Figure 3: Alternating-voltage and droop/active power control block diagram

where the diagonal sub-matrices are the equivalent impedance models of the converter on either sides, and the off-diagonal sub-matrices are the transfer gains that predict the interaction between the $\mathrm{AC}$ and $\mathrm{DC}$ sides of the converter. It is important to remark that, either one of the off-diagonal sub-matrices can be adopted to predict the interaction between the AC and DC subnetworks as seen from the PCC and DC buses of a specific converter $i$.

\subsubsection{AC and DC Droop/Active Power Controlled VSCs}

The standard proposed DC droop voltage structure depicted in Fig. 3 can be applied as an alternative control strategy for autonomous compensation in the DC grid. Note that constant active power controller can be obtained by setting droop gain to zero; whereas DC voltage can be prioritized with a high but finite droop gain [26]. Following similar procedures as for the constant DC voltage controlled converter and noting that for the droop strategy

$$
\left\{\begin{array}{l}
P_{t o t}^{*}=P^{*}+R_{d c}\left(V_{d c}^{*}-V_{d c}\right) \\
\Delta P_{t o t}^{*}=\Delta P^{*}=-R_{d c} \Delta V_{d c}
\end{array}\right.
$$

where the outer DC voltage and power references are assumed constant and $R_{d c}$ is the droop constant, which are passed on to the inner-loop where in linearized form

$$
\Delta i_{c d}^{*}=K_{p}(s)\left(\Delta P^{*}-\Delta P\right)
$$

where $K_{p}(s)$ is the active power compensator, $\Delta P$ and $\Delta P^{*}$ from (5) and (15) respectively, and the resulting $\Delta i_{c d}^{*}$ is substituted into the linearized equation of converter modulation voltages as done previously. The AC voltage controller is similar to that modelled previously. Likewise, the states, and the state-space coefficients are constructed as previously and the impedance model is of a similar structure to (14).

For the sake of uniformity between impedance and admittance models, the converter equivalent impedance model can be derived as

$$
\mathbf{Z}_{a c i}(s)=\left(\begin{array}{ll}
Y_{d d i}(s) & Y_{d q i}(s) \\
Y_{q d i}(s) & Y_{q q i}(s)
\end{array}\right)^{-1}
$$

Fig. 4 illustrates the circuit representation of equation (14), showing how the impedances connect on each side of a VSC at an arbitrary terminal $i . H_{c l}^{v}(s)$ and $H_{c l}^{i}(s)$ are the closed-loop DC voltage and current reference to output transfer functions. These are eliminated after linearization at an operating point. 


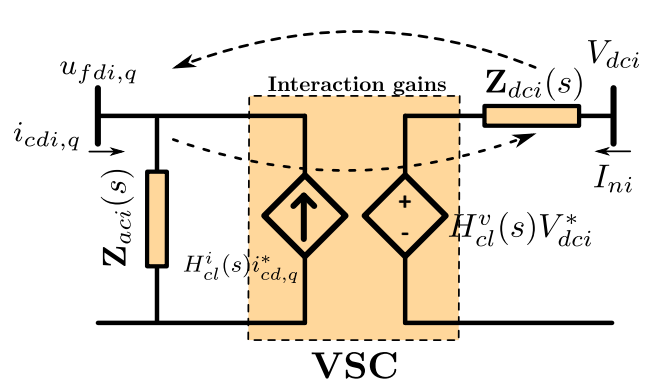

Figure 4: Circuit equivalent of impedance model of a VSC
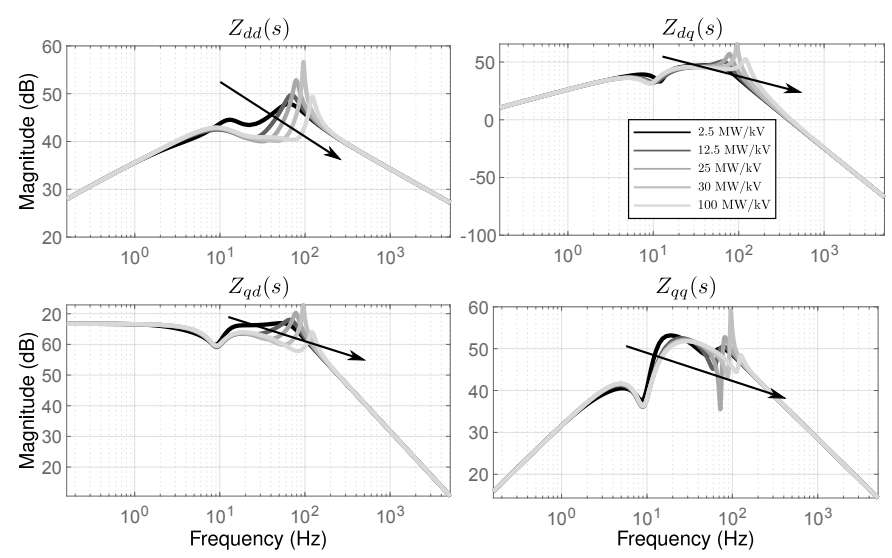

Figure 5: Impact of varying droop gains on the frequency response of the equivalent AC impedance of the VSC

For the grid connected converter in this paper with data presented in Table 1, Fig. 5 shows the frequency responses of the converter AC side equivalent impedance to variation in droop gain. It can be observed that at the converter-level, increasing the droop gain initially results to increasing magnitude of resonances, and shift of resonance frequency to the high frequency region. As droop gain is further increased, then, resonance magnitude reduces. This is the result of the VSC having a behaviour closer to DC voltage controlled as previously mentioned for a high but finite droop gain. This can be inferred from (15), by considering two limiting values of the droop gain $R_{d c} \rightarrow 0$ and $R_{d c} \rightarrow \infty$ (but finite). In the former, the VSC behaves as an active power controlled converter and in the latter, behaves as a DC voltage controlled converter[26]. However, this may not be desired if a specific droop response $\left(0 \ll R_{d c} \ll \infty\right)$ is required.

On the DC side, the frequency response to the same variation as previously is shown in Fig. 6. It can be seen that the DC side shows a uniform behaviour. For increasing droop gain, the steady-state gain simply reduces; this is equivalent to a lower steady-state deviation. Additionally, on the DC side, although a similar frequency shifting is observed compared to the $\mathrm{AC}$ side, the increasing droop gain steadily reduces the magnitude of resonance. This again can be explained in a similar manner as previously. Therefore, in summary, this suggest a fairly low droop gain is desired on the $\mathrm{AC}$ side and a slightly higher gain on the DC side. Hence, proper operation will require a trade-off

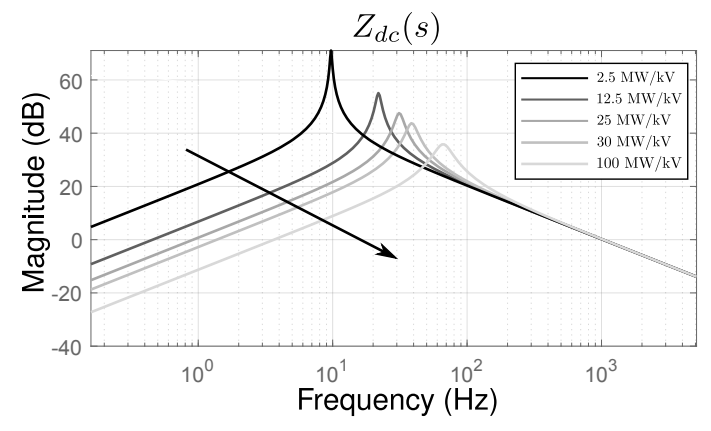

Figure 6: Impact of varying droop gains on the frequency response of the equivalent DC impedance of the VSC
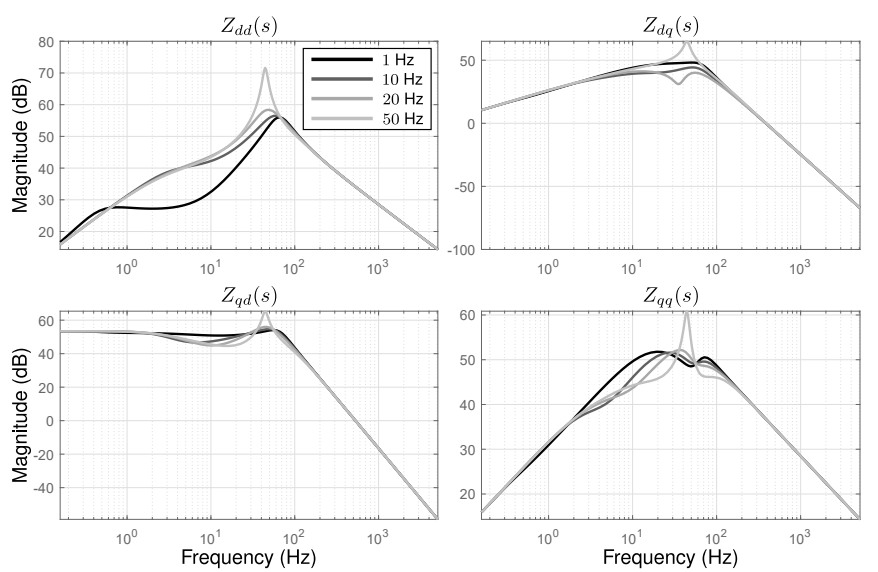

Figure 7: Impact of PLL bandwidth on the frequency response of the equivalent $\mathrm{AC}$ impedance of the $\mathrm{VSC}$

considering the entire system responses (on both AC and DC sides).

To verify the impact of the PLL on converter-level impedances, Fig. 7 shows the frequency responses of the AC impedance for varying PLL bandwidths. As can be observed, for the $d$-axis impedance, increasing the PLL bandwidth increases the magnitude response, especially in the low frequency region $(<10 \mathrm{~Hz})$. With a faster PLL at $50 \mathrm{~Hz}$, a significant increase in the resonance magnitude is observed at a frequency quite close to the fundamental that will destabilize the interconnected system. For the $q$ axis impedance, except for the PLL bandwidth at $50 \mathrm{~Hz}$, the impact of PLL bandwidth is negligible. A similar conclusion can be made for the cross-coupled impedances on either axes. Hence, a PLL bandwidth below $50 \mathrm{~Hz}$ can be adopted. As for the DC side impedance, the parameters of the PLL do not directly influence the frequency responses as acknowledged in [25]. It is also important to remark that the shown frequency responses are dependent on operating point and more so with power direction inversion or rectification. In this paper, the grid connected $\mathrm{VSC}$ is assumed to be in inverter operation.

\subsection{Transmission Lines and Cables}

Transmission lines and cables are one of the most important subsystems of any transmission system. Particu- 


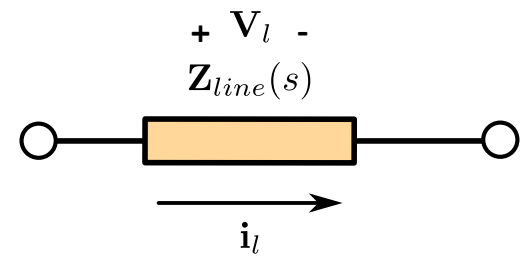

Figure 8: Equivalent circuit of a transmission line or cable

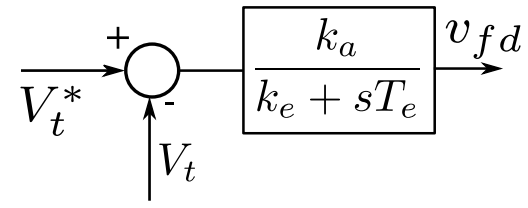

Figure 9: AVR/exciter block diagram

larly, any interaction between components are facilitated by the network dictated by the lines and/or cables. In this paper, all lines and cables are represented by their $\Pi$ equivalents in Laplace. On the $\mathrm{AC}$ side, each line must be represented by its $d q$ pair including the cross-coupling given as

$$
\mathbf{Z}_{\text {line }}(s)=\left(\begin{array}{cc}
R_{l}+s L_{l} & -\omega_{1} L_{l} \\
\omega_{1} L_{l} & R_{l}+s L_{l}
\end{array}\right)
$$

where $R_{l}$ and $L_{l}$ are the line resistance and inductance respectively and $\omega_{1}$ is the fundamental frequency; the admittance equivalent can be applied as well. Fig. 8 depicts the equivalent circuit representation of a transmission line or cable.

\section{4. dq Modelling of Synchronous Generators}

In this paper, a simplified model of the synchronous generator is implemented. The main focus of the study is the influence of the excitation system on the input-output behaviour of the generator that connects via a network to a converter also controlling the voltage and power at its bus. Therefore, speed variations, damper winding effect, and load compensator for AVR/exciter loop of synchronous generation are neglected for simplicity.

The open-loop state-space equations for the synchronous generator in $d q$-frame can be expressed as [27]

$$
\Delta \dot{x}_{g}=\mathbf{L}_{m}^{-1} \mathbf{R}_{m} \Delta x_{g}+\mathbf{L}_{m}^{-1} \Delta V_{g}
$$

where $\mathbf{L}_{m}, \mathbf{R}_{m}$ are the inductance and resistance matrices consisting of stator $d, q$-axes, and field components, with states and input vectors as

$$
\begin{aligned}
& \Delta x_{g}=\left(\Delta i_{d}, \Delta i_{q}, \Delta i_{f d}\right)^{T} \\
& \Delta V_{g}=\left(\Delta v_{d}, \Delta v_{q}, \Delta v_{f d}\right)^{T}
\end{aligned}
$$

$\Delta v_{d}, \Delta v_{q}$, and $\Delta v_{f d}$ are the stator and field voltages respectively; $i_{d}, i_{q}, i_{f d}$ are the stator and field currents respectively on corresponding axes. An AVR/exciter combination consisting of DC exciter and regulator as shown
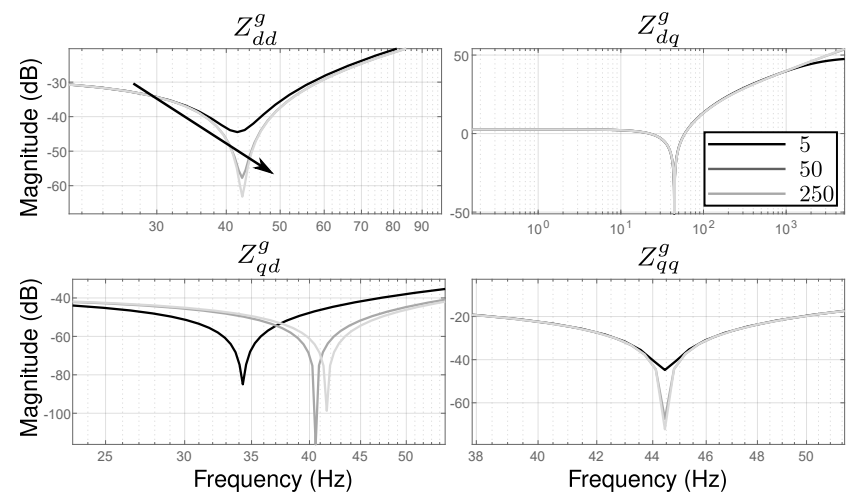

Figure 10: Frequency response of generator equivalent impedance with increasing amplifier gain

in Fig. 9 is implemented [28]. The total transfer function of the AVR/exciter can be expressed as

$$
K_{a v r}(s)=\frac{k_{a}}{k_{e}+s T_{e}}
$$

where $k_{a}$ is the regulator gain, $k_{e}$ is the exciter gain, and $T_{e}$ is the exciter time constant, $V_{t}$ is the terminal voltage given as

$$
V_{t}=\sqrt{v_{d}^{2}+v_{q}^{2}}
$$

The linearized exciter and regulator expression can be written as

$$
\Delta v_{f d}=K_{a v r}(s)\left(\Delta V_{t}^{*}-\Delta V_{t}\right)=-K_{a v r}(s) \Delta V_{t}
$$

where terminal voltage reference $V_{t}^{*}$ is constant. Eliminating $\Delta v_{f d}$ as an input from (20) and applying (2), the input-output impedance model of the synchronous generator can be obtained as

$$
\left(\begin{array}{c}
\Delta v_{d} \\
\Delta v_{q}
\end{array}\right)=\underbrace{\left(\begin{array}{cc}
Z_{d d}^{g}(s) & Z_{d q}^{g}(s) \\
Z_{q d}^{g}(s) & Z_{q q}^{g}(s)
\end{array}\right)}_{\mathbf{Z}_{c l}^{g}(s)}\left(\begin{array}{c}
\Delta i_{d} \\
\Delta i_{q}
\end{array}\right)
$$

where $\mathbf{Z}_{c l}^{g}(s)$ is the output impedance model of the synchronous generator on generator $d q$-axis with influence of AVR/exciter included. Fig. 10 shows the frequency response of the synchronous generator with AVR/exciter modelled for increasing amplifier gain showing the potential frequency shifting that could occur. However, the true impact of AVR amplifier is most observed on interconnection with the network of lines and network topology.

\section{5. $d q$ Modelling of Capacitive Components}

The filter capacitor connected at the VSC PCC and other capacitive components can be modelled by the equivalent admittance matrix expressed as

$$
\mathbf{Y}_{f}(s)=\left(\begin{array}{cc}
s C_{f} & -\omega_{1} C_{f} \\
\omega_{1} C_{f} & s C_{f}
\end{array}\right)
$$

where $C_{f}$ is the filter capacitance. The equivalent impedance model can be also be utilized. 


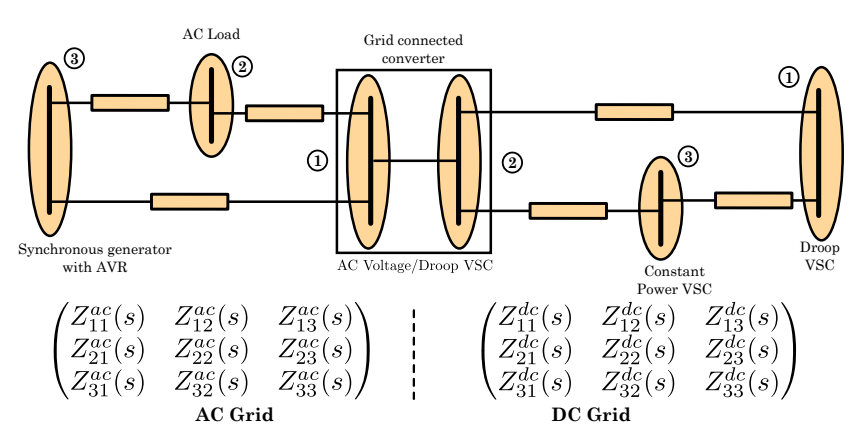

Figure 11: Interconnection of subsystems and components

\section{Interconnection of Impedance Models of Sub- systems}

Considering that the synchronous generator and $\mathrm{AC}$ side of the converter are on separate $d q$ frames, it is very important to transform both impedance models to a common reference frame before interconnection; in this paper, the converter is chosen as the reference frame and transformation is done according to

$$
\left(\begin{array}{l}
d^{c} \\
q^{c}
\end{array}\right)=\left(\begin{array}{cc}
\cos \left(\theta_{c}\right) & \sin \left(\theta_{c}\right) \\
-\sin \left(\theta_{c}\right) & \cos \left(\theta_{c}\right)
\end{array}\right)\left(\begin{array}{l}
d \\
q
\end{array}\right)
$$

where $d q^{c}$ are the expression in the chosen global reference frame (in this case, the converter) and $\theta_{c}$ is the difference in angle between the global reference frame and the original reference frame. After transformation to a common reference frame, an interconnection according to the network topology is made as illustrated in Fig. 11, where the equivalent impedance of each subsystem (oval shapes) is interconnected, showing the corresponding control modes of each subsystem. The grid connected VSC consists of two oval shapes that denote the equivalent models on each $\mathrm{AC}$ and DC sides. The AC sides of Other VSCs are not of interest, hence they are represented by their DC equivalents.

\subsection{The $Z_{\text {bus }}$ Impedance Aggregation}

The $\mathbf{Z}_{\text {bus }}$ is simply the impedance matrix widely applied in power system analysis [29]. The elements of the $\mathbf{Z}_{\text {bus }}$ matrix is synonymous with the Thévenin equivalents as seen from each terminal and between terminals and can be crudely obtained by inverting the admittance equivalents. Clearly, the entire hybrid AC/DC grid has been explicitly represented by impedance equivalents and manipulation can be done considering the physical connotation. Particularly, the stability and interaction between any two interface in the system can be studied as required, while any grid or component can be simplified as necessary.

\subsubsection{AC Equivalent Matrix}

The AC equivalent impedance matrix can be obtained by aggregating the impedance equivalents of each subsys-
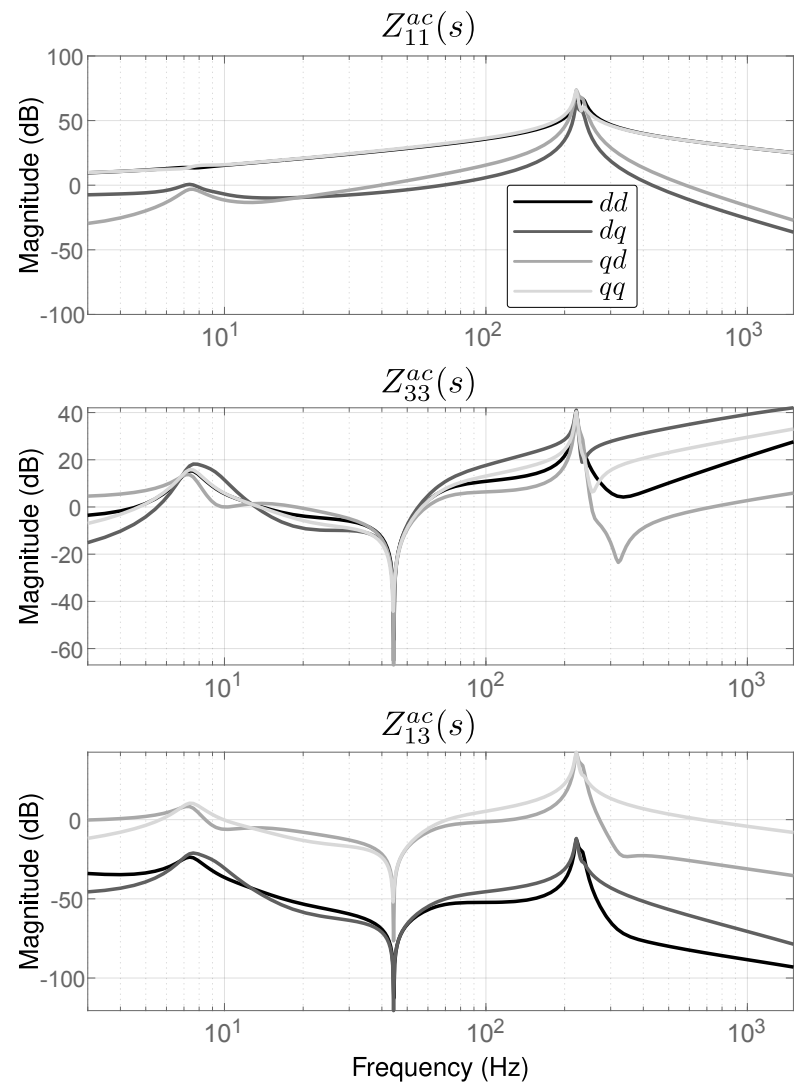

Figure 12: Frequency response of equivalent impedances at buses 1 and 3

tem following Fig. 11, which is expressed as

$$
\mathbf{Z}_{c l}^{a c}(s)=\left(\begin{array}{ccc}
Z_{11}^{a c}(s) & Z_{12}^{a c}(s) & Z_{13}^{a c}(s) \\
Z_{21}^{a c}(s) & Z_{22}^{a c}(s) & Z_{23}^{a c}(s) \\
Z_{31}^{a c}(s) & Z_{32}^{a c}(s) & Z_{33}^{a c}(s)
\end{array}\right)
$$

such that,

$$
\Delta \mathbf{V}_{a c}=\mathbf{Z}_{c l}^{a c}(s) \Delta \mathbf{I}_{a c}
$$

where $\Delta \mathbf{I}_{a c}$ and $\Delta \mathbf{V}_{a c}$ are the vector of AC network currents and bus voltages respectively during disturbances. Each element in (27) is a $2 \times 2$ matrix and each dictate the dynamic responses at each terminal and between terminals. The diagonal elements are the impedances seen by each device connected to each terminal and they show the contribution of every other device in the system to the response observed at a particular terminal. The frequency responses of system-level impedances at the controlled buses 1 (converter) and 3 (generator), and the transfer impedance between them for droop and AVR gains given in Tables 1 and 2 is shown in Fig. 12. At the converter terminal, the converter AC impedance has been reshaped and new resonances introduced around $220 \mathrm{~Hz}$ and a slightly damped resonance around $7 \mathrm{~Hz}$. Whereas on the generator side, the $220 \mathrm{~Hz}$ resonance is clear and the $7 \mathrm{~Hz}$ resonance has a higher magnitude as seen from this bus. 


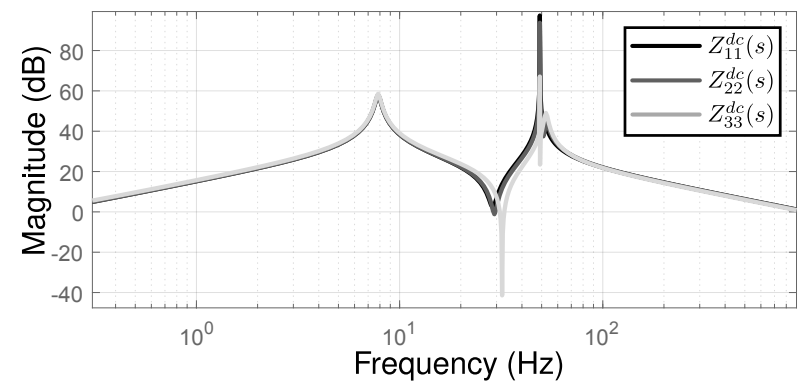

Figure 13: Frequency response of terminal impedances of the DC grid

\subsubsection{Equivalent Matrix}

The DC equivalent matrix can be obtained in a similar manner as the AC grid. The DC system-level equivalent impedances can be expressed as

$$
\mathbf{Z}_{c l}^{d c}(s)=\left(\begin{array}{ccc}
Z_{11}^{d c}(s) & Z_{12}^{d c}(s) & Z_{13}^{d c}(s) \\
Z_{21}^{d c}(s) & Z_{22}^{d c}(s) & Z_{23}^{d c}(s) \\
Z_{31}^{d c}(s) & Z_{32}^{d c}(s) & Z_{33}^{d c}(s)
\end{array}\right)
$$

such that,

$$
\Delta \mathbf{V}_{d c}=\mathbf{Z}_{c l}^{d c}(s) \Delta \mathbf{I}_{n}
$$

where $\Delta \mathbf{V}_{d c}$ and $\Delta \mathbf{I}_{n}$ are the network bus voltages and currents respectively, during disturbances. Fig. 13 shows the frequency response of diagonal elements of the DC equivalent matrix showing clearly the resonances seen from each terminal. It can be observed that, all terminals in the DC grid share resonances around 7.8 and $49.5 \mathrm{~Hz}$, with a higher magnitude at the latter resonance. Further, although slightly shifted from each other, observe that the low frequency resonance at $7.8 \mathrm{~Hz}$ is shared with the low frequency resonance in the $\mathrm{AC}$ grid at $7 \mathrm{~Hz}$. This is an interaction point between the $\mathrm{AC}$ and DC grids. However, the near-synchronous resonance dominates the DC grid dynamics compared to the low frequency counterpart. Other distinct frequencies can be observed within the DC grid slightly shifted from each other.

\section{Analysis and Case Study}

The studied system is shown in Fig. 1. The matrix of equations (27) and (29) apply to each of AC and DC systems respectively. As the frequency responses of each subnetwork shown in Figs. 12 and 13, the matrices consist of individual dynamics of each subnetwork and shared dynamics through the grid connected VSC. To simplify the system, on the AC sides responses are shown for buses 1 and 3 are relevant as they are the only controlled buses.

\subsection{Interconnection of Hybrid System}

The simulation responses for the AC side of the interconnected hybrid AC/DC grid is shown in Fig. 14 with the fast Fourier transform (FFT) plot. Highly distorted waveforms with both high and low frequency resonances
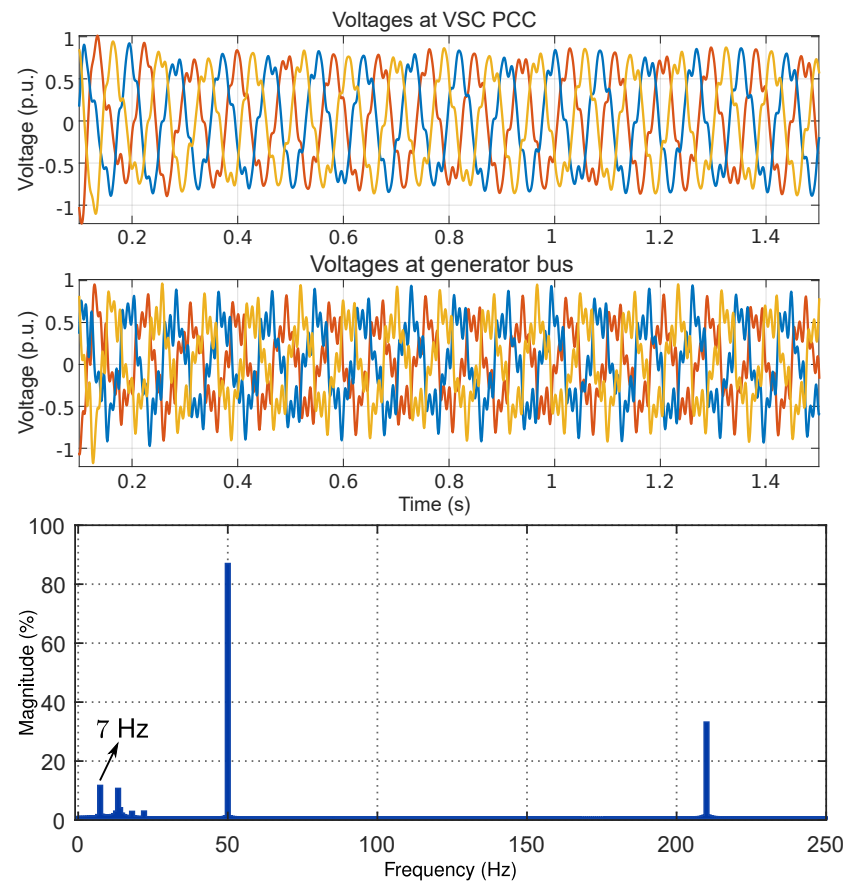

Figure 14: Simulation of AC voltages at VSC PCC and generator bus, with FFT of the voltages showing the impacts of generator regulator gain

can be observed matching the observed frequencies in Fig. 12 as the FFT shows. On the DC side, Fig. 15 depicts the corresponding DC voltage responses with the FFT plot. Likewise, it shows the DC voltage responses are dominated by the near-synchronous resonance, whereas, the low frequency resonance is well-damped as the FFT shows. In summary, it can be seen that the high frequency resonance on the AC side is related to the AC grid; whereas the nearsynchronous resonance is related to the DC grid, and the $7 \mathrm{~Hz}$ is shared. Although as expected, the $7 \mathrm{~Hz}$ is welldamped on the DC side.

The shown responses are not acceptable as clearly the synchronous generator was not considered in the choice of droop gain in the DC grid. Therefore, either the AVR/exciter gain or DC droop gain must be reduced to minimize the impact of the low frequency resonance around $5-10 \mathrm{~Hz}$. In a similar manner, the dominant resonance in the DC system must also be managed by simply utilizing a damping scheme based on the identified frequencies that trims the dominant peak of the $\mathrm{DC} \mathbf{Z}_{c l}^{d c}(s)$ matrix.

\subsection{Impacts of DC Droop Gain}

For step change of 0.3 p.u. of active power in the MTDC grid at the constant power VSC at $t=3 \mathrm{~s}$, the impact of high droop gain is shown in Figs. 17 and 18. It can be seen that the step change resulted to loss of stability in both the $\mathrm{AC}$ and $\mathrm{DC}$ grids. Therefore, a reduction in droop gain is first applied, and use of a damper for the $49.5 \mathrm{~Hz}$ resonance in the DC grid which is not related to the AC grid. The structure of the damper is shown in 

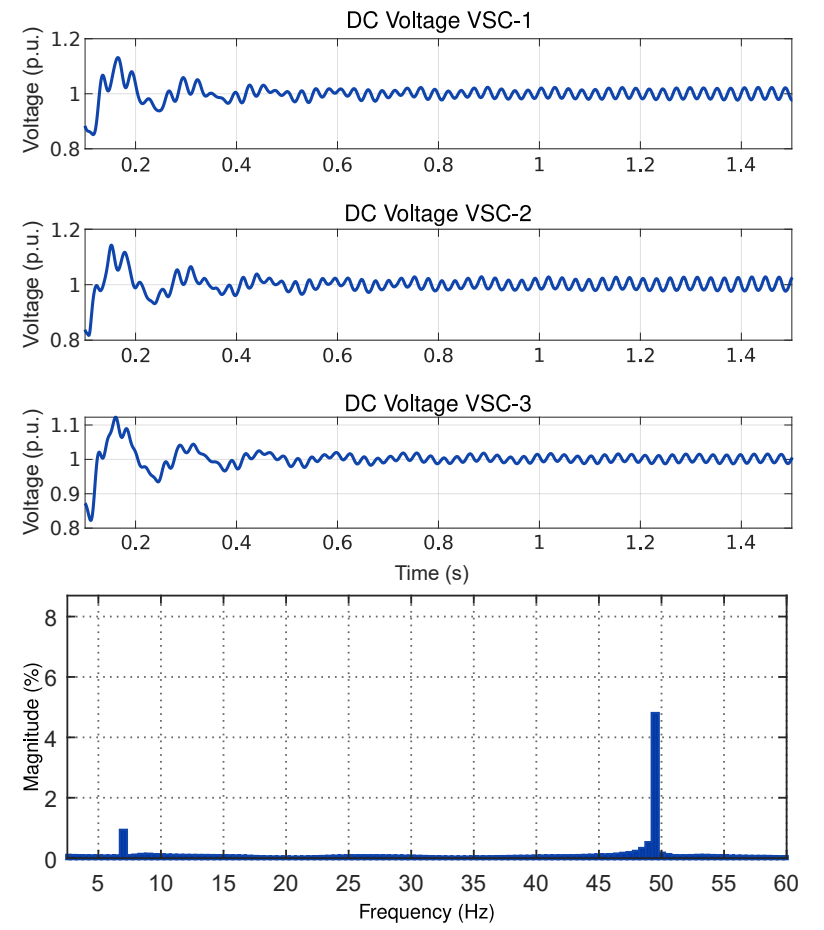

Figure 15: Simulation of DC voltages with the FFT plot showing the impact of generator regulator gain and DC side oscillations

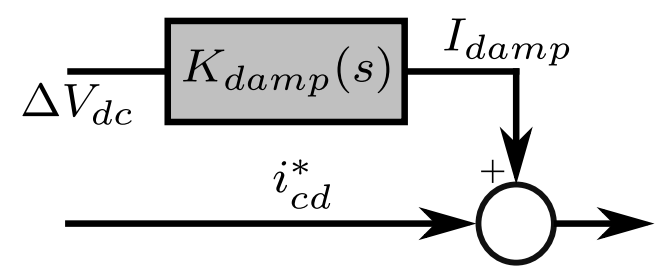

Figure 16: DC side damper structure

Fig. 16 where the bus voltage deviation is passed through a bandpass filter $K_{d a m p}(s)$ and the output is added to the reference current from outer-loops and sent to the innercurrent controller (Fig. 2). The bandpass filter is realized with a centre frequency around $48 \mathrm{~Hz}$ with bus voltage deviation as input, with a transfer function given as in (31). It is important to remark that since the $49.5 \mathrm{~Hz}$ resonance is related to the DC grid, the damper can be installed at any controllable terminal in the DC grid. For a reduction in droop gain $(5 \mathrm{MW} / \mathrm{kV})$ and exciter regulator gain $\left(K_{a}=50\right)$, Fig. 19 depicts the AC voltages for the same step change showing an acceptable performance on the AC side.

$$
K_{\text {damp }}(s)=\frac{\left(\omega_{0} / Q\right) s}{s^{2}+\left(\omega_{0} / Q\right) s+\omega_{0}^{2}}
$$

where $\omega_{0}$ is the centre frequency, and $Q$ is the quality factor.

Fig. 20 shows a comparison of the responses of the DC voltages for a reduction in droop gain with the synchronous generator connected and the default droop gain at that terminal when the generator is disconnected. Particularly, it is shown how that despite the reduced droop
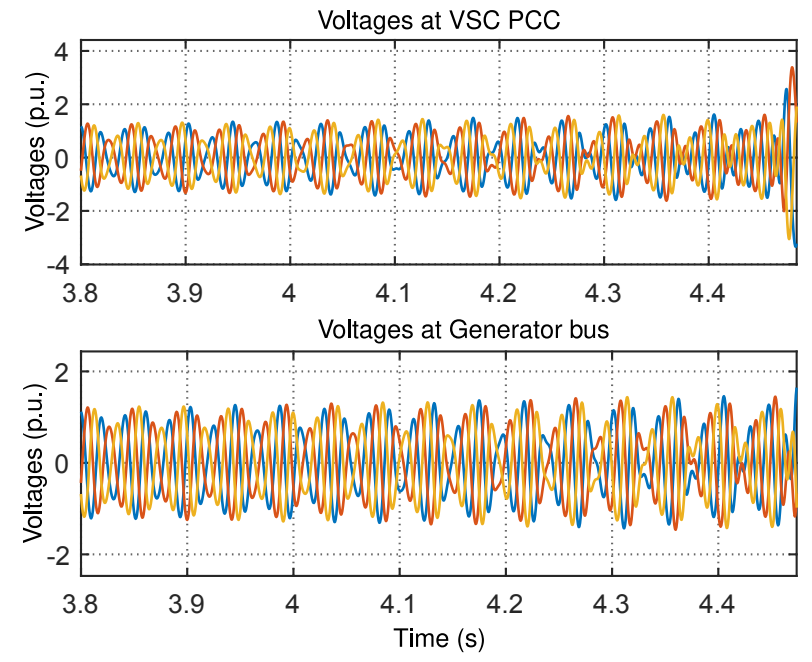

Figure 17: Time domain simulation of AC voltages at VSC PCC and generator bus showing the impacts of DC side droop gain

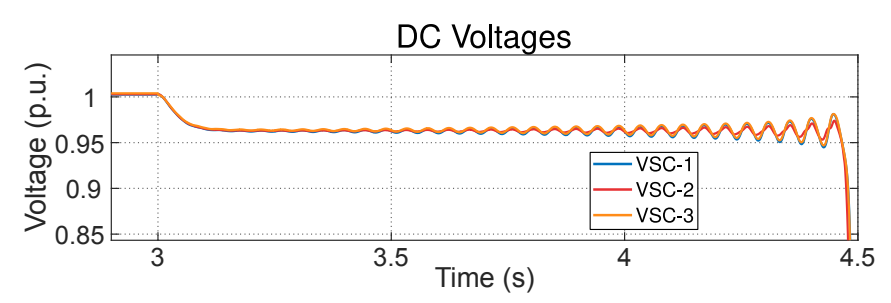

Figure 18: Time domain simulation of DC voltages showing the impacts of DC side droop gain
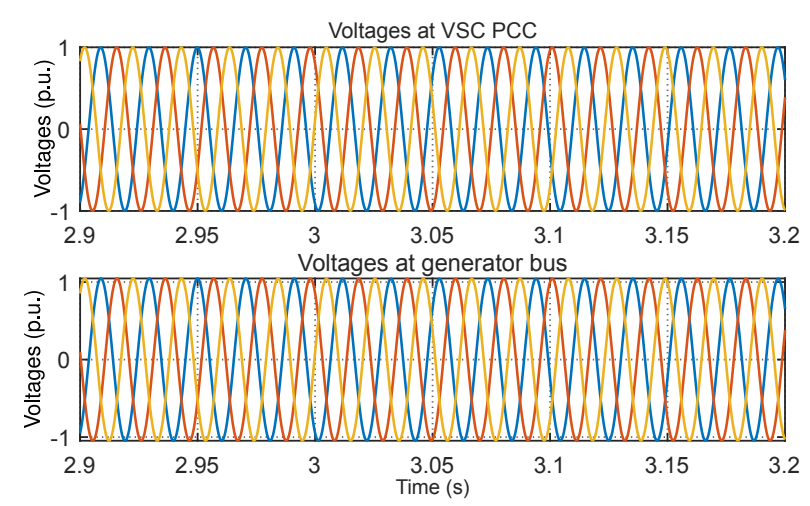

Figure 19: Time domain simulation of AC voltages at VSC PCC and generator bus showing the impacts of reduced DC side droop gain

gain improving the dynamics on the $\mathrm{AC}$ side, it results to a higher deviation in voltages compared to if a higher droop gain is utilized without a synchronous generator. Moreover, it shows that the DC grid is stable without the synchronous generator connected. This demonstrates the required trade-offs in performance required of hybrid $\mathrm{AC} / \mathrm{DC}$ grids due to conflicting requirements.

\section{Conclusions}

This paper extends the derivations of subsystem-level impedance equivalents to system-level equivalents in a 

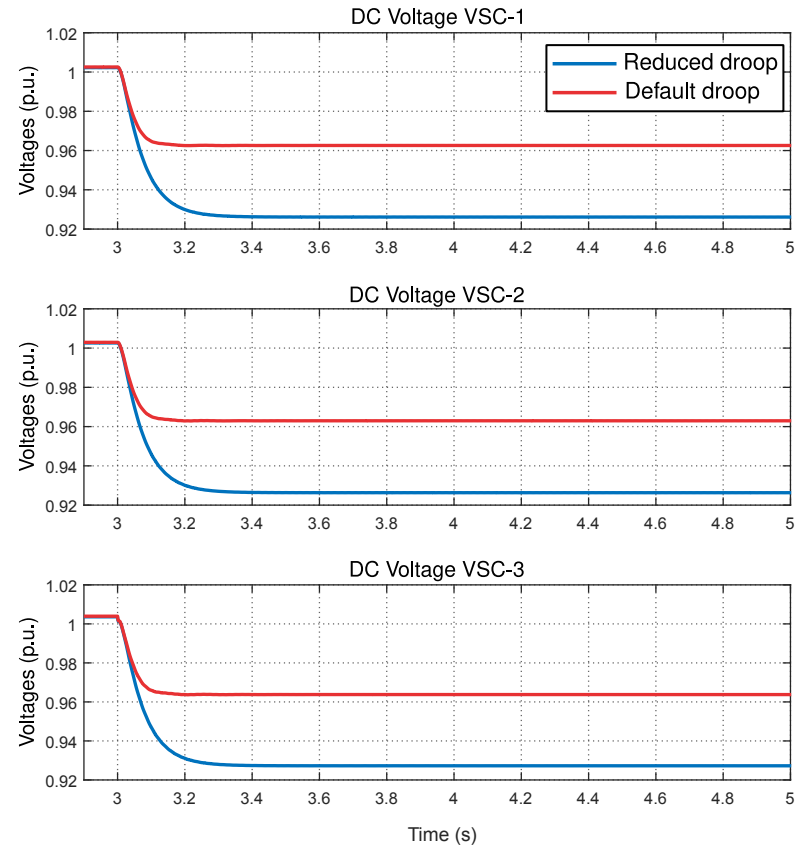

Figure 20: Time domain simulation of DC voltages showing the impacts of reduced DC side droop gain and the default gain with the AC generator is disconnected

manner that decouples the AC and DC subnetworks of a hybrid network. As a case, the interaction between a VSC interconnected on both sides, with a synchronous generator and its excitation system on the AC side was studied. It was shown how this method is capable of highlighting the individual dynamics of each subnetwork and the shared dynamics between each that reflects the interaction between both. Overall, the impact of relevant controller parameters of the key subsystems were shown and their effect on interactions demonstrated. In the end, it was shown that a compromise in controller parameters of active subsystems were required to achieve an acceptable performance when interconnected. To summarize, results show the potential of this method to analyse interactions with interconnected grid and components, while utilizing existing frequency domain tools. However, in order to improve the scalability of impedance models for network-scale analysis in a truly large-scale AC/DC grid, there may be need to further reduce the order and structure of models.

Table 1: DC Grid Parameters

\begin{tabular}{lc}
\hline Parameter & Value \\
\hline Rated Power & $800 \mathrm{MVA}$ \\
Reference DC voltage & $400 \mathrm{kV}$ \\
$C_{d c}$ & $150 \mu \mathrm{F}$ \\
$L_{c a b}$ & $2.6 \mathrm{mH} / \mathrm{km}$ \\
$C_{c a b}$ & $0.1908 \mu \mathrm{F} / \mathrm{km}$ \\
$R_{c a b}$ & $0.0141 \Omega / \mathrm{km}$ \\
Cable length $(12,13,23)$ & $70,150,100 \mathrm{~km}$ \\
Droop gain & $10 \mathrm{MW} / \mathrm{kV}$ \\
\hline
\end{tabular}

Table 2: AC Grid Parameters

\begin{tabular}{lc}
\hline Parameter & Value \\
\hline Nominal ac voltage & $\pm 220 \mathrm{kV}(\mathrm{p}-\mathrm{p})$ \\
$L_{\text {line }}$ (+ve seq.) & $0.0011 \mathrm{mH} / \mathrm{km}$ \\
$R_{\text {line }}$ (+ve seq.) & $0.028 \Omega / \mathrm{km}$ \\
Cable length $(12,13,23)$ & $123,200,115 \mathrm{~km}$ \\
$L$ & $42 \mathrm{mH}$ \\
$C_{f}$ & $10 \mu \mathrm{F}$ \\
$R$ & $0.242 \Omega$ \\
Stator $\left(L_{d}, L_{q}\right)$ & $4.9825 \mathrm{mH}, 4.85 \mathrm{mH}$ \\
Stator $R_{s}$ & $0.0031 \Omega$ \\
$k_{a}, k_{e}, T_{e}$ & $200,1,1.25 \mathrm{~s}$ \\
\hline
\end{tabular}

\section{AppendixA.}

\section{Transformation Matrices}

The linearized inverse transformation matrices between grid and converter reference frames for converter currents and voltages can be expressed as

$$
\begin{aligned}
\mathbf{T}^{i} & =\left(\begin{array}{ccc}
\cos \theta_{p}^{0} & \sin \theta_{p}^{0} & -i_{c d}^{0} \sin \theta_{p}^{0}+i_{c q}^{0} \cos \theta_{p}^{0} \\
-\sin \theta_{p}^{0} & \cos \theta_{p}^{0} & -i_{c d}^{0} \cos \theta_{p}^{0}-i_{c q}^{0} \sin \theta_{p}^{0}
\end{array}\right) \\
\mathbf{T}^{v} & =\left(\begin{array}{ccc}
\cos \theta_{p}^{0} & \sin \theta_{p}^{0} & -u_{f d}^{0} \sin \theta_{p}^{0}+u_{f q}^{0} \cos \theta_{p}^{0} \\
-\sin \theta_{p}^{0} & \cos \theta_{p}^{0} & -u_{f d}^{0} \cos \theta_{p}^{0}-u_{f q}^{0} \sin \theta_{p}^{0}
\end{array}\right),
\end{aligned}
$$

whereas, the transformation matrix between converter and grid frame for the converter voltages can be expressed as

$$
\mathbf{T}=\left(\begin{array}{ccc}
\cos \theta_{p}^{0} & -\sin \theta_{p}^{0} & -u_{c d}^{c 0} \sin \theta_{p}^{0}-u_{c q}^{c 0} \cos \theta_{p}^{0} \\
\sin \theta_{p}^{0} & \cos \theta_{p}^{0} & u_{c d}^{c 0} \cos \theta_{p}^{0}-u_{c q}^{c 0} \sin \theta_{p}^{0}
\end{array}\right)
$$

PLL Transfer Functions

$$
\begin{aligned}
& H_{d \theta}(s)=\frac{K_{p l l}(s) \sin \theta_{p}^{0}}{s-K_{p l l}(s)\left(u_{f d}^{0} \cos \theta_{p}^{0}+u_{f q}^{0} \sin \theta_{p}^{0}\right)} \\
& H_{q \theta}(s)=\frac{K_{p l l}(s) \cos \theta_{p}^{0}}{s-K_{p l l}(s)\left(u_{f d}^{0} \cos \theta_{p}^{0}+u_{f q}^{0} \sin \theta_{p}^{0}\right)}
\end{aligned}
$$

\section{Acknowledgements}

This work was financially supported by the European Union's Horizon 2020 research and innovation programme under Marie-Sklodowska-Curie action INCITE (grant agreement No. 675318). 


\section{References}

[1] E. M. Lightner, S. E. Widergren, An orderly transition to a transformed electricity system, IEEE Transactions on Smart Grid 1 (1) (2010) 3-10. doi:10.1109/TSG . 2010.2045013.

[2] N. Flourentzou, V. G. Agelidis, G. D. Demetriades, Vsc-based hvdc power transmission systems: An overview, IEEE Transactions on Power Electronics 24 (3) (2009) 592-602. doi: 10.1109/TPEL. 2008. 2008441.

[3] C. Meyer, M. Hoing, A. Peterson, R. W. D. Doncker, Control and design of dc grids for offshore wind farms, IEEE Transactions on Industry Applications 43 (6) (2007) 1475-1482. doi:10.1109/TIA.2007.908182.

[4] F. Schettler, H. Huang, N. Christl, Hvdc transmission systems using voltage sourced converters design and applications, in: 2000 Power Engineering Society Summer Meeting (Cat. No.00CH37134), Vol. 2, 2000, pp. 715-720 vol. 2. doi:10.1109/ PESS. 2000.867439.

[5] D. V. Hertem, M. Ghandhari, Multi-terminal vsc hvdc for the european supergrid: Obstacles, Renewable and Sustainable Energy Reviews 14 (9) (2010) 3156 - 3163. doi:http: //dx.doi.org/10.1016/j.rser.2010.07.068.

URL http://www.sciencedirect.com/science/article/pii/ S1364032110002480

[6] W. Lu, B. Ooi, Multi-terminal HVDC as enabling technology of premium quality power park, in: IEEE Power Engineering Society Winter Meeting, 2002., Vol. 2, 2002, pp. 719-724 vol.2. doi:10.1109/PESW. 2002.985098.

[7] T. Ackermann, T. Prevost, V. Vittal, A. J. Roscoe, J. Matevosyan, N. Miller, Paving the way: A future without inertia is closer than you think, IEEE Power and Energy Magazine 15 (6) (2017) 61-69. doi:10.1109/MPE. 2017.2729138.

[8] N. R. Chaudhuri, R. Majumder, B. Chaudhuri, J. Pan, Stability analysis of VSC MTDC grids connected to multimachine AC systems, IEEE Transactions on Power Delivery 26 (4) (2011) 2774-2784. doi:10.1109/TPWRD.2011.2165735.

[9] M. Ndreko, M. Popov, J. L. Rueda-Torres, M. A. M. M. van der Meijden, Impact of offshore wind and conventional generation outages on the dynamic performance of ac-dc transmission systems, in: PowerTech, 2015 IEEE Eindhoven, 2015, pp. 1-6. doi:10.1109/PTC.2015.7232397.

[10] R. Eriksson, J. Beerten, M. Ghandhari, R. Belmans, Optimizing DC voltage droop settings for AC/DC system interactions, IEEE Transactions on Power Delivery 29 (1) (2014) 362-369. doi:10.1109/TPWRD . 2013.2264757.

[11] L. Shen, M. Barnes, R. Preece, J. V. Milanovic, K. Bell, M. Belivanis, The effect of VSC-HVDC control on ac system electromechanical oscillations and DC system dynamics, IEEE Transactions on Power Delivery 31 (3) (2016) 1085-1095. doi : 10.1109/TPWRD. 2015.2414824.

[12] M. Amin, A. Rygg, M. Molinas, Impedance-based and eigenvalue based stability assessment compared in vsc-hvdc system, in: 2016 IEEE Energy Conversion Congress and Exposition (ECCE), 2016, pp. 1-8. doi:10.1109/ECCE. 2016.7855185.

[13] J. Sun, Impedance-based stability criterion for grid-connected inverters, IEEE Transactions on Power Electronics 26 (11) (2011) 3075-3078. doi:10.1109/TPEL.2011.2136439.

[14] L. Harnefors, M. Bongiorno, S. Lundberg, Input-admittance calculation and shaping for controlled voltage-source converters, IEEE Transactions on Industrial Electronics 54 (6) (2007) 3323-3334. doi:10.1109/TIE. 2007.904022.

[15] L. Xu, L. Fan, Z. Miao, Dc impedance-model-based resonance analysis of a vsc-hvdc system, IEEE Transactions on Power Delivery 30 (3) (2015) 1221-1230. doi:10.1109/TPWRD. 2014. 2367123.

[16] M. Cespedes, J. Sun, Impedance modeling and analysis of grid-connected voltage-source converters, IEEE Transactions on Power Electronics 29 (3) (2014) 1254-1261. doi:10.1109/TPEL. 2013. 2262473.

[17] X. Wang, F. Blaabjerg, W. Wu, Modeling and analysis of harmonic stability in an ac power-electronics-based power system,
IEEE Transactions on Power Electronics 29 (12) (2014) 64216432. doi:10.1109/TPEL.2014.2306432.

18] M. Amin, M. Molinas, J. Lyu, X. Cai, Impact of power flow direction on the stability of vsc-hvdc seen from the impedance nyquist plot, IEEE Transactions on Power Electronics 32 (10) (2017) 8204-8217. doi:10.1109/TPEL . 2016. 2608278.

[19] B. Wen, D. Dong, D. Boroyevich, R. Burgos, P. Mattavelli, Z. Shen, Impedance-based analysis of grid-synchronization stability for three-phase paralleled converters, IEEE Transactions on Power Electronics 31 (1) (2016) 26-38. doi:10.1109/TPEL. 2015. 2419712.

[20] M. Amin, M. Molinas, Understanding the origin of oscillatory phenomena observed between wind farms and hvdc systems, IEEE Journal of Emerging and Selected Topics in Power Electronics 5 (1) (2017) 378-392. doi:10.1109/JESTPE. 2016. 2620378.

[21] J. Lv, P. Dong, G. Shi, X. Cai, H. Rao, J. Chen, Subsynchronous oscillation of large dfig-based wind farms integration through mmc-based hvdc, in: 2014 International Conference on Power System Technology, 2014, pp. 2401-2408. doi : 10.1109/POWERCON. 2014.6993945.

[22] K. N. B. M. Hasan, K. Rauma, A. Luna, J. I. Candela, P. Rodrguez, Harmonic compensation analysis in offshore wind power plants using hybrid filters, IEEE Transactions on Industry Applications 50 (3) (2014) 2050-2060. doi:10.1109/TIA.2013. 2286216.

[23] L. Wang, X. Xie, Q. Jiang, H. Liu, Y. Li, H. Liu, Investigation of ssr in practical dfig-based wind farms connected to a series-compensated power system, IEEE Transactions on Power Systems 30 (5) (2015) 2772-2779. doi:10.1109/TPWRS. 2014. 2365197.

[24] A. Bayo-Salas, J. Beerten, D. Van Hertem, Analytical methodology to develop frequency-dependent equivalents in networks with multiple converters, in: 2017 IEEE Manchester PowerTech, 2017, pp. 1-6. doi:10.1109/PTC. 2017.7980938.

[25] A. J. Agbemuko, J. L. Domnguez-Garca, E. Prieto-Araujo, O. Gomis-Bellmunt, Dynamic modelling and interaction analysis of multi-terminal vsc-hvdc grids through an impedancebased approach, International Journal of Electrical Power \& Energy Systems 113 (2019) 874 - 887. doi:https://doi.org/ 10.1016/j.ijepes.2019.06.029.

[26] T. M. Haileselassie, K. Uhlen, Impact of dc line voltage drops on power flow of mtdc using droop control, IEEE Transactions on Power Systems 27 (3) (2012) 1441-1449. doi:10.1109/TPWRS. 2012.2186988.

[27] P. Krause, O. Wasynczuk, S. D. Sudhoff, S. Pekarek, Analysis of electric machinery and drive systems, Vol. 75, John Wiley \& Sons, 2013.

[28] P. Kundur, N. J. Balu, M. G. Lauby, Power system stability and control, Vol. 7, McGraw-hill New York, 1994.

[29] J. J. Grainger, W. D. Stevenson Jr., Power system analysis, McGraw-Hill International Editions, 1994. 